0.64 ) and sulfonylurea (MD: $-14.21, \mathrm{CI}:-22.86$ to -5.56 ). SGLT2 $\mathrm{i}$ showed the greatest reduction in BMI, being superior to sulfonylurea (MD: $-1.67, \mathrm{CI}:-3.09$ to -0.25 ) and standard of care (MD: 3.45, CI: 1.24 to 5.66). SGLT2i showed the greatest reduction of $\mathrm{LDL}$ and $\mathrm{TG}$ and the greatest increase of HDL.

Conclusions SGLT2i, GLP-1RA and PPAR $\gamma$ agonists are viable choices for the management of NAFLD in diabetic patients. SGLT2 $\mathrm{i}$ was found to be most efficacious for fibrosis and BMI reduction, GIP1-RA for steatosis reduction and PPAR $\gamma$ agonists for lipid modulation and should be considered over sulfonylurea for second-line therapy.

\section{IDDF2021-ABS-0102 RETROSPECTIVE ANALYSIS OF CHARACTERISTICS, INDICATIONS AND OUTCOMES OF ERCP IN A TERTIARY REFERRAL CENTER OF SAUDI ARABIA}

${ }^{1}$ Laeeque Ahmed*, ${ }^{2}$ Ahmed Basalim, ${ }^{3}$ Nawaf Alwagdani, ${ }^{2}$ Bsaim Altirkistani, ${ }^{4}$ Ziyad Albassam, ${ }^{5}$ Mohammed Almatrafi, ${ }^{3}$ Faisal Alrubaei, ${ }^{1}$ Adnan Alzanbagi. ${ }^{1}$ King Abdullah Medical City Makkah, Saudi Arabia; ${ }^{2}$ King Saud Bin Abdul Aziz University, Jeddah, Saudi Arabia; ${ }^{3}$ College of Medicine, Taif University, Taif, Saudi Arabia; ${ }^{4}$ King Abdul Aziz University, Jeddah, Saudi Arabia; 5 Umm Al Qura University, Makkah, Saudi Arabia

\subsection{6/gutjnl-2021-IDDF.90}

Background Endoscopic Retrograde Cholangiopancreatography (ERCP) is an advanced endoscopic procedure that is an essential tool in the management of pancreaticobiliary diseases. In the Arab world, there is limited data available on the indications and outcomes of ERCP. Therefore, we aimed to report the characteristics of patients, indications, and outcomes of ERCP from a tertiary referral center in Saudi Arabia.

Methods We retrospectively looked at ERCP procedures done at a tertiary referral center covering the western region of Saudi Arabia from August 2018 till July 2020. Data were collected from the hospital's electronic patient record and endoscopy database.

Results Of 1001 ERCP's performed, full data was available on 712 procedures on 581 patients that were included in the final analysis. Mean age was 53.1. 404 (56.7\%) were female. All ERCPs were performed by certified consultants, with therapeutic intention. Sphincterotomy was performed in 563/581 (96.9\%) patients who underwent first-ever ERCP. The commonest indication of ERCP was confirmed or suspected choledocholithiasis $(52.6 \%)$, followed by replacement or removal of a biliary stent $(15.7 \%), 55(7.7 \%)$ for suspected ascending cholangitis, $54(7.5 \%)$ for severe acute biliary pancreatitis, and $15(2 \%)$ for suspected sphincter of Oddi dysfunction. The commonest finding among all patients was choledocholithiasis in $57.9 \%$, debris in $15.2 \%$, and biliary stricture in $14.8 \%$. The commonest complication was pancreatitis 22/712 (3.1\%) followed by post-sphincterotomy bleeding 16/712 (2.2\%) and perforation $9 / 712(1.2)$. Bleeding was controlled by endoscopic intervention in $4 / 16(25 \%)$ and $1 / 16(6.2 \%)$ patient underwent surgery. $2 / 712(0.28 \%)$ patients had procedure-related mortality, one secondary to post-procedure pulmonary embolism and the other because of complicated perforation. The deeper CBD cannulation rate was 98.2\% (570/581)

Conclusions Our study results revealed that ERCP performed in Saudi Arabia has similar indications and findings as compared to international literature. ERCP is successful in achieving the therapeutic objective with complication rates consistent with published literature.

\section{IDDF2021-ABS-0112 SAFETY OF CONTINUING ANTICOAGULATION DURING ENDOSCOPIC VARICEAL LIGATION IN CIRRHOSIS PATIENTS: A SYSTEMATIC REVIEW AND META-ANALYSIS}

${ }^{1}$ Wei Xuan Tay*, ${ }^{1}$ Gerald Low Jun Teck, ${ }^{1}$ Jing Hong Loo, ${ }^{1}$ Le Shaun Ang, ${ }^{2}$ Yu Jun Wong. ${ }^{1}$ Yong Loo Lin School of Medicine, National University of Singapore, Singapore; ${ }^{2}$ Changi General Hospital, Singapore

\subsection{6/gutjnl-2021-IDDF.91}

Background Endoscopic variceal ligation (EVL) is a common procedure for primary and secondary prevention of variceal bleeding in cirrhosis patients. As portal vein thrombosis is associated with a higher risk of variceal bleeding and decompensation in cirrhosis patients, anticoagulation may be considered in these patients. However, it is uncertain if continuing anticoagulation during EVL may significantly increase the risk of bleeding. To address this gap, we performed a systematic review and meta-analysis to evaluate the safety of continuing anticoagulation during EVL. The primary outcome was the bleeding risk following EVL. Secondary outcomes were mortality following EVL and length of stay (LOS).

Methods We performed a systematic search of 4 electronic databases (PubMed/MEDLINE, EMBASE, Cochrane library, and ClinicalTrial.gov) up to May 21, 2021 using both free text and $\mathrm{MeSH}$ terms. We included all studies that reported the 4-week rebleeding rate in cirrhosis patients during EVL, regardless of study design, language, publication types or status. The pooled relative risk (RR), mean difference (MD) and $95 \%$ confidence interval $(95 \% \mathrm{CI})$ of study outcomes were estimated using Review Manager in a random-effect model.

Results A total of six studies (5,102 subjects, 1,028 received anticoagulation) fulfilled our inclusion criteria. The mean (standard deviation) MELD and Child-Pugh score were 18 (5.5) and 7 (1.6), respectively. Follow-up duration ranged from 6 to 196 weeks. The overall pooled risk of bleeding and mortality was $8.2 \%$ (95\%CI: $6.6 \%-10.0 \%)$ and $8.6 \%$ (95\%CI: 4.2\%-15.3\%), respectively. Mean number of bands deployed per session was similar between the anticoagulation and control group. Continuation of anticoagulants during EVL was not associated with a higher pooled risk of bleeding (RR: 0.90, 95\%CI: 0.71-1.15, $I^{2}=0 \%$ ) and mortality (RR: 1.16, 95\%CI: 0.22-6.19) following EVL. However, continuing anticoagulation was associated with a shorter LOS (3.9 days, 95\% CI: -7.35 to -0.48 days, $I^{2}=0 \%$ ). All studies had a low risk of bias except for one.

Conclusions In cirrhosis patients undergoing EVL, continuing anticoagulation was safe without a significant increase in bleeding and mortality.

\section{IDDF2021-ABS-0116 SURGICAL TREATMENT FOR NON- COLORECTAL NON-NEUROENDOCRINE LIVER METASTASES: A SYSTEMATIC REVIEW AND META-ANALYSIS}

Kelvin $\mathrm{Ng}^{*}$, Nicole Cheng, Hon-Ting Lok, Charing Chong, John Wong, Kit-Fai Lee. The Chinese University of Hong Kong, Hong Kong

\subsection{6/gutjnl-2021-IDDF.92}

Background Liver-only metastasis from primary tumor elsewhere is common. Hepatectomy for colorectal or neuroendocrine liver metastases have favourable survival outcomes and 
acceptable surgical morbidities and mortalities. However, the role of hepatectomy for non-colorectal non-neuroendocrine liver metastases (NCNNLM) is still controversial. This study aims to perform a systematic review and meta-analysis of important clinical outcomes after hepatectomy for NCNNLM. Methods Electronic search of Pubmed and Embase databases were performed to identify all relevant published studies. Patient demographics, surgical procedures, peri-operative and survival outcomes were documented. Primary end-points were 3- and 5- year overall survival (OS) and disease-free survival (DFS). Secondary end-points were post-operative morbidity, 30-day mortality and prognostic factors associated with survival.

Results A total of 161 studies were identified involving patients with NCNNLM undergone hepatectomy. Thirty-six studies including 3827 patients met the inclusion criteria. Fourteen studies analysed NCNNLM as a whole group, while
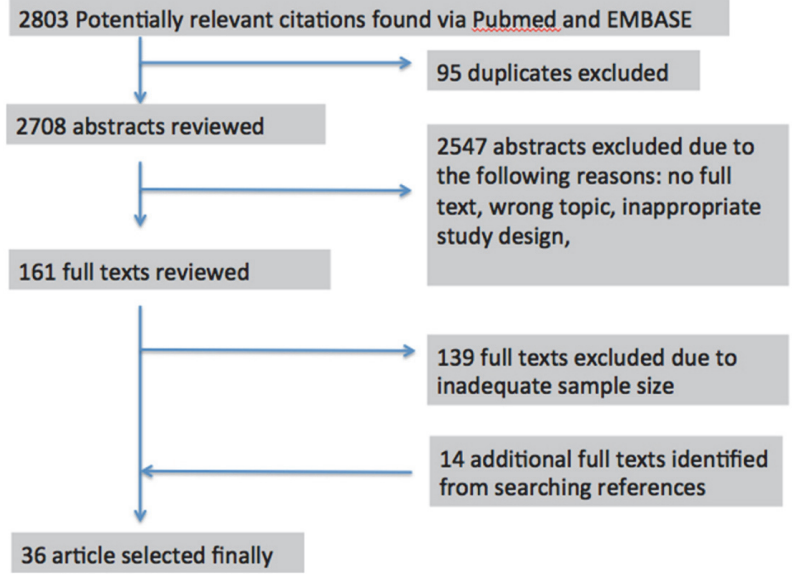

Abstract IDDF2021-ABS-0116 Figure 1

$\begin{array}{lr}\text { Study } & \text { Number of 3-Year Overall Survival } \\ & \\ \text { Kamel et al 2011 } & 50 \\ \text { Marudanayagam et al 2011 } & 31 \\ \text { Duan et al 2012 } & 59 \\ \text { Groeschl et al 2012 } & 210 \\ \text { Takemura et al 2012 } & 32 \\ \text { Takemura et al 2013 } & 80 \\ \text { Kinoshita et al 2014 } & 107 \\ \text { Slotta et al 2014 } & 43 \\ \text { Guner et al 2015 } & 28 \\ \text { Tiberio et al 2015 } & 7 \\ \text { Oki et al 2016 } & 48 \\ \text { Schiergens et al 2016 } & 82 \\ \text { Tiberio et al 2016 } & 21 \\ \text { Li et al 2017 } & 19 \\ \text { Li et al 2017 } & 49 \\ \text { Markar et al 2017 } & 33 \\ \text { Song et al 2017 } & 46 \\ \text { Labgaa et al 2018 } & 114 \\ \text { Aghayan et al 2019 } & 27\end{array}$

Random effects model

Heterogeneity. $I^{2}=78 \%, \tau^{2}=0.1210, p<0.01$

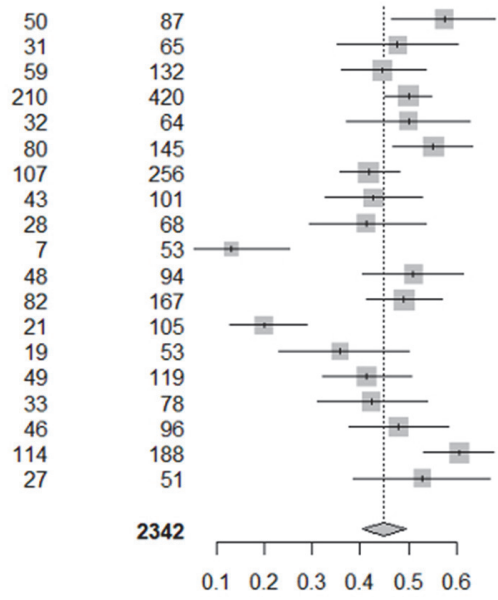

Proportion $\quad 95 \%-\mathrm{Cl}$ Weight

$0.57[0.46 ; 0.68]-5.2 \%$ $0.48[0.35 ; 0.60] \quad 4.8 \%$ $0.45[0.36 ; 0.54] \quad 5.8 \%$ $0.50[0.45 ; 0.55] \quad 6.7 \%$ $0.50[0.37 ; 0.63] \quad 4.8 \%$ $0.55[0.47 ; 0.63] \quad 5.9 \%$ $0.42[0.36 ; 0.48] \quad 6.4 \%$ $0.43[0.33 ; 0.53] \quad 5.4 \%$ $0.41[0.29 ; 0.54] \quad 4.8 \%$ $0.13[0.05 ; 0.25] \quad 3.1 \%$ $0.51[0.41 ; 0.62] \quad 5.4 \%$ $0.49[0.41 ; 0.57] \quad 6.0 \%$ $0.20[0.13 ; 0.29] \quad 4.9 \%$ $0.36[0.23 ; 0.50] \quad 4.3 \%$ $0.41[0.32 ; 0.51] \quad 5.6 \%$ $0.42[0.31 ; 0.54] \quad 5.0 \%$ $0.48[0.38 ; 0.58] \quad 5.4 \%$ $0.61[0.53 ; 0.68] \quad 6.1 \%$ $0.53[0.38 ; 0.67] \quad 4.4 \%$

$0.45[0.40 ; 0.50] 100.0 \%$

\section{Abstract IDDF2021-ABS-0116 Figure 2}

\begin{tabular}{|c|c|c|c|c|c|c|}
\hline Number of 5-Y & vival & Total Patient & & Proportion & $95 \%-\mathrm{Cl}$ & Weight \\
\hline Staehler et al 2010 & 55 & 88 & +1 & 0.62 & {$[0.52 ; 0.73]$} & $3.3 \%$ \\
\hline Bresadola et al 2011 & 17 & 56 & & 0.30 & {$[0.19 ; 0.44]$} & $2.9 \%$ \\
\hline Kamel et al 2011 & 36 & 87 & 1 & 0.41 & {$[0.31 ; 0.52]$} & $3.3 \%$ \\
\hline Marudanayagam et al 2011 & 17 & 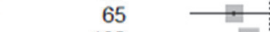 & - & 0.26 & {$[0.16 ; 0.39]$} & $3.0 \%$ \\
\hline Duan et al 2012 & 39 & 132 & & 0.30 & {$[0.22 ; 0.38]$} & $3.5 \%$ \\
\hline Groeschl et al 2012 & 130 & 420 & & 0.31 & {$[0.27 ; 0.36]$} & $3.9 \%$ \\
\hline Niu et al 2012 & 18 & 60 & & 0.30 & {$[0.19 ; 0.43]$} & $3.0 \%$ \\
\hline Takemura et al 2012 & 24 & 64 & + & 0.38 & {$[0.26 ; 0.50]$} & $3.1 \%$ \\
\hline Takemura et al 2013 & 59 & 145 & 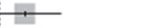 & 0.41 & {$[0.33 ; 0.49]$} & $3.6 \%$ \\
\hline Aizawa et al 2014 & 14 & 1 & & 0.19 & {$[0.11 ; 0.30]$} & $2.9 \%$ \\
\hline Faries et al 2014 & 17 & $\ldots$ & - & 0.29 & {$[0.18,0.43]$} & $2.9 \%$ \\
\hline Kinoshita et al 2014 & 80 & 256 & & 0.31 & {$[0.26 ; 0.37]$} & $3.7 \%$ \\
\hline Slotta et al 2014 & 30 & 101 & - & 0.30 & {$[0.21 ; 0.40]$} & $3.3 \%$ \\
\hline Brudvik et al 2015 & 81 & 146 & $\longrightarrow$ & 0.55 & {$[0.47 ; 0.64]$} & $3.6 \%$ \\
\hline Guner et al 2015 & 20 & 68 & - & 0.29 & {$[0.19 ; 0.42]$} & $3.1 \%$ \\
\hline Hoffman et al 2015 & 63 & 150 & 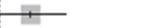 & 0.42 & {$[0.34 ; 0.50]$} & $3.6 \%$ \\
\hline Maeda et al 2015 & 18 & 59 & - & 0.31 & {$[0.19 ; 0.44]$} & $3.0 \%$ \\
\hline Martel et al 2015 & 30 & 52 & 1 & 0.58 & {$[0.43 ; 0.71]$} & $3.0 \%$ \\
\hline Tiberio et al 2015 & 5 & $53 \longrightarrow$ & & 0.09 & {$[0.03 ; 0.21]$} & $2.0 \%$ \\
\hline Oki et al 2016 & 40 & 94 & + & 0.43 & {$[0.32 ; 0.53]$} & $3.4 \%$ \\
\hline Sadot et al 2016 & 62 & 167 & & 0.37 & {$[0.30 ; 0.45]$} & $3.6 \%$ \\
\hline Tiberio et al 2016 & 14 & $105 \multimap$ & & 0.13 & {$[0.07 ; 0.21]$} & $2.9 \%$ \\
\hline Li et al 2017 & 11 & $53 \longrightarrow$ & & 0.21 & {$[0.11 ; 0.34]$} & $2.7 \%$ \\
\hline Li et al 2017 & 30 & 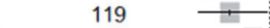 & & 0.25 & {$[0.18 ; 0.34]$} & $3.4 \%$ \\
\hline Markar et al 2017 & 24 & $\longrightarrow$ & & 0.31 & {$[0.21 ; 0.42]$} & $3.2 \%$ \\
\hline Song et al 2017 & 21 & 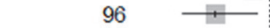 & & 0.22 & {$[0.14 ; 0.31]$} & $3.2 \%$ \\
\hline Goumard et al 2018 & 62 & 126 & $\longrightarrow$ & 0.49 & {$[0.40 ; 0.58]$} & $3.5 \%$ \\
\hline Holzner et al 2018 & 57 & 100 & $\longrightarrow$ & 0.57 & {$[0.47 ; 0.67]$} & $3.4 \%$ \\
\hline Labgaa et al 2018 & 90 & 188 & 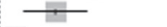 & 0.48 & {$[0.41 ; 0.55]$} & $3.7 \%$ \\
\hline Sim et al 2018 & 30 & 78 & + & 0.38 & {$[0.28 ; 0.50]$} & $3.3 \%$ \\
\hline Aghayan et al 2019 & 19 & 51 & 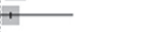 & 0.37 & {$[0.24 ; 0.52]$} & $2.9 \%$ \\
\hline \multirow{2}{*}{$\begin{array}{l}\text { Random effects model } \\
\text { Heterogeneity: } I^{2}=84 \%, \tau^{2}=0.2190, p<0.01\end{array}$} & & 3389 & & \multirow{2}{*}{\multicolumn{2}{|c|}{$0.35[0.31 ; 0.39]$}} & $100.0 \%$ \\
\hline & & 0.102 & & & & \\
\hline
\end{tabular}


Number of 3-Year

Disease-free Survival Total Patient

Kinoshita et al 2014

Guner et al 2015

Hoffman et al 2015

Oki et al 2016

83
21
54
27

Fixed effect model

Heterogeneity: $I^{2}=0 \%, \tau^{2}=0, p=0.68$

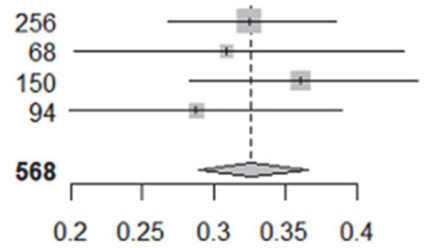

Proportion $\quad 95 \%-\mathrm{Cl}$ Weight

$0.32[0.27 ; 0.39] \quad 45.1 \%$

$0.31[0.20 ; 0.43] \quad 11.7 \%$

$0.36[0.28 ; 0.44] \quad 27.8 \%$

$0.29[0.20 ; 0.39] \quad 15.5 \%$

$0.33[0.29 ; 0.37] 100.0 \%$

\section{Abstract IDDF2021-ABS-0116 Figure 4}

Study
Kinoshita et al 2014
Slotta et al 2014
Brudvik et al 2015
Hoffman et al 2015
Maeda et al 2015
Oki et al 2016
Goumard et al 2018

\section{Number of 5-Year} Disease-free Survival Total Patient

Random effects model

Heterogeneity: $I^{2}=61 \%, \tau^{2}=0.0611, p=0.02$

77
25
52
44
17
26
19

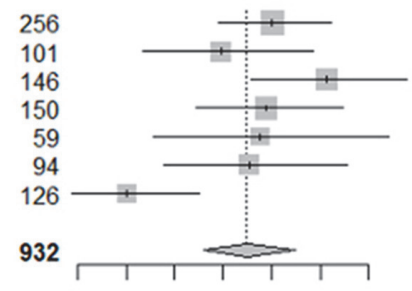

$\begin{array}{lllllll}0.1 & 0.15 & 0.2 & 0.25 & 0.3 & 0.35 & 0.4\end{array}$

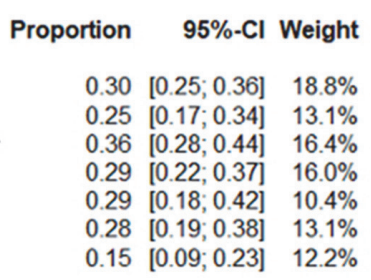

$0.27[0.23 ; 0.32] 100.0 \%$

\section{Abstract IDDF2021-ABS-0116 Figure 5}

32 studies described tumors with a specified primary, with gastric cancer being the most common. (IDDF2021-ABS-0116 Figure 1) Pooled data showed that the 3- and 5-year OS were $45 \% \quad(95 \%$ CI $0.40-0.50)$ and $35 \%$ (95\% CI $0.31-0.19)$, (IDDF2021-ABS-0116 Figure 2, IDDF2021-ABS-0116 Figure 3) respectively, whereas the 3- and 5-year DFS were $33 \%$ (95\% CI 0.29-20.37) and 27\% (95\% CI 0.23-0.32), respectively (IDDF2021-ABS-0116 Figure 4, IDDF2021-ABS-0116 Figure 5). The postoperative morbidity rate was $24 \%(95 \%$ CI $0.20-0.29)$, whereas the 30 -day mortality was $2 \%(95 \%$ CI 0.01-0.03). Gastric cancer with liver metastasis had 3-year and 5 -year OS of $39 \%(95 \%$ CI $0.32-0.46)$ and $25 \%(95 \%$ CI 0.20-0.31).

Conclusions Hepatectomy for NCNNLM may achieve favourable survival outcomes, with low surgical morbidities and mortalities in selected patients.

\section{IDDF2021-ABS-0118 FACTORS ASSOCIATED WITH THE PRESENCE OF NON-ALCOHOLIC FATTY LIVER DISEASE (NAFLD) DETECTED IN JAPANESE ADULTS UNDERGOING HEALTH CHECK-UPS}

Toh Yoon Ezekiel Wong. Hiroshima Kyoritsu Hospital, Japan

\subsection{6/gutjnl-2021-IDDF.93}

Background Non-alcoholic fatty liver disease (NAFLD) and steatohepatitis (NASH) is a growing cause of cirrhosis and hepatocellular carcinoma (HCC) in Japan. We examined the prevalence of NAFLD in a Japanese cohort undergoing health check-ups and factors associated with its presence.

Methods This cross-sectional study included 130 non-drinking adults who participated in health check-ups with abdominal ultrasonography option during a 3 month period. Age, gender, BMI, waist circumference, ALT, $\gamma$-GTP, triglyceride (TG), HDL and HbA1c levels were analysed as variables possibly associated with the presence of NAFLD.

\begin{tabular}{lll}
\multicolumn{2}{l}{ Abstract IDDF2021-ABS-0118 Table 1 } & \\
\hline Variables/Biomarkers & Adjusted odds Ratio $(95 \% \mathrm{Cl})$ & P value \\
\hline Age (years) & $0.98(0.93$ to 1.03$)$ & 0.39 \\
Gender (male vs. female) & $0.85(0.32$ to 2.25$)$ & 0.75 \\
BMl (kg/m2) & $1.38(1.17$ to 1.64$)$ & 0.0001 \\
Triglyceride (mg/dL) & $1.01(1.00$ to 1.01$)$ & 0.07 \\
HbA1c (\%) & $1.71(0.97$ to 3.01$)$ & 0.06 \\
\hline
\end{tabular}

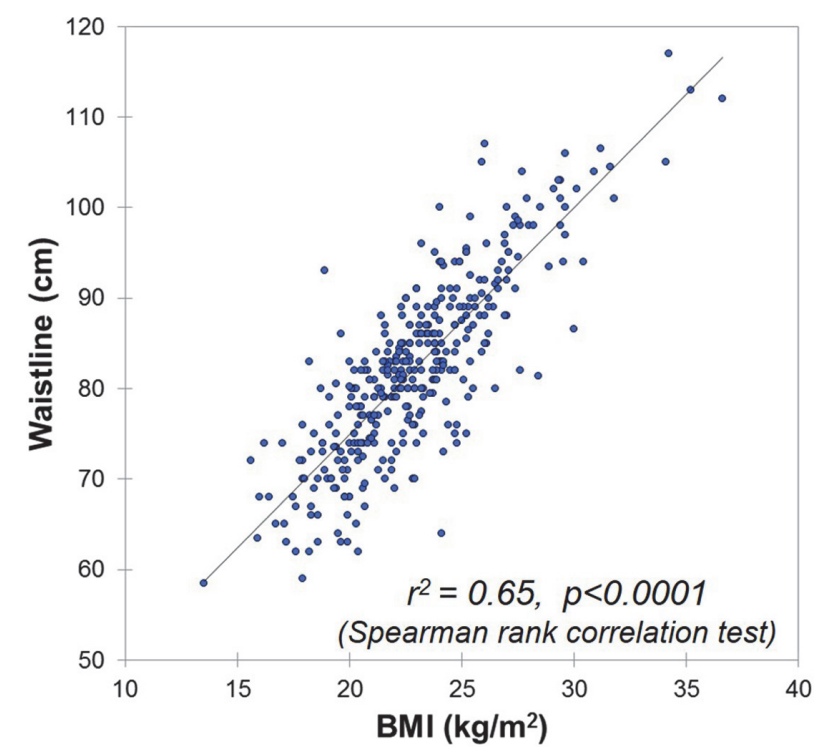

Abstract IDDF2021-ABS-0118 Figure 1 